\title{
Flow and heat transfer over a longitudinal circular cylinder moving in parallel or reversely to a free stream
}

\author{
T.-Y. Na, Dearborn, Michigan, and I. Pop, Cluj, Romania
}

(Received April 24, 1995)

Summary. Steady laminar boundary layer flow and heat transfer over a thin longitudinal isothermal circular cylinder moving in a flowing stream has been studied in this paper. The cases in which the cylinder is moving in the same (parallel) or in the opposite (reverse) direction to the free stream are considered. The transformed nonsimilar boundary layer equations are solved numerically using the Keller-box method for some values of the curvature parameter, the Prandtl number and relative velocity parameter. The results show that the velocity and temperature distributions as well as the coefficients of skin friction and the local Nusselt number are appreciably affected by the relative velocity parameter.

\section{Nomenclature}

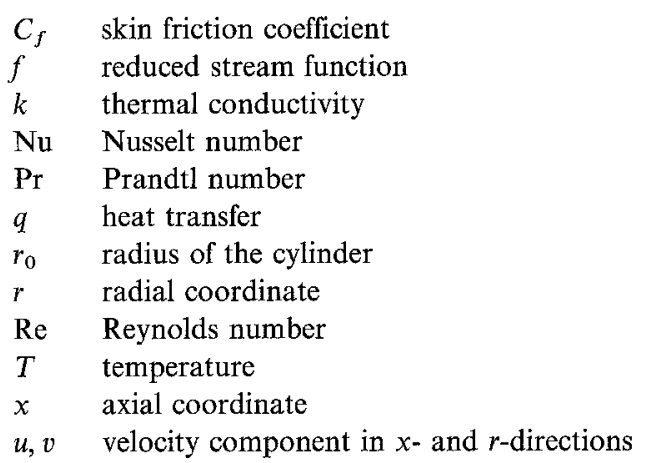

\footnotetext{
Greek symbols

$\xi \quad$ curvature parameter

$\eta \quad$ pseudo-similarity variable

$\theta$ dimensionless temperature

$\lambda \quad$ relative velocity parameter

$\mu \quad$ dynamic viscosity

$v \quad$ kinematic viscosity

$\tau \quad$ skin friction

$\varrho \quad$ density

$\psi \quad$ stream function
}

\section{Superscript}

partial differentiation with respect to $\eta$ 
Subscripts

w condition at the wall

$\infty \quad$ ambient condition

\section{Introduction}

The laminar boundary layer flow and heat transfer along the outer surface of a static circular cylinder in axial flow has been extensively studied in the past by Seban and Bond [1], Kelly [2], Glauert and Lighthill [3], Stewartson [4], and Okamura [6], Cebeci [7], Curle [8], Lin and Shih [9] and Sawchuk and Zamir [10]. However, in many practical engineering systems both the cylinder and the ambient fluid are moving in parallel or reversely. Examples are in the manufacture of fibres, in glass and polymer industries. The boundary layer flow for a continuously moving cylinder in axial direction has also been studied by several authors. Sakiadis [11] produced the first study regarding the boundary layer behavior on a cylindrical surface moving in a quiescent fluid. The work of Sakiadis [11] was extended by Rotte and Beek [12], Lin and Shih [9], Karnis and Pechoc [13], Choi [14], Pop et al. [15] and Eswara and Nath [16].

The purpose of this paper is to study the general forced convection flow and heat transfer over a longitudinal thin circular cylinder, which is moving in the same (parallel) or in the opposite (reverse) direction to the free stream. In order to analyze the effect of both the moving cylinder and the free stream on the boundary layer new variables are introduced, which are the combination of the classical ones for the static and for the moving cylinder, respectively. The transformed equations are non-similar and they are solved numerically using the Keller-box method introduced by Keller and Cebeci [17]. Sufficient details to provide essential features of the velocity and temperature distributions from which estimates of the skin friction coefficients and local Nusselt number can be obtained. It should be mentioned that the present analysis is more general than any previous investigation.

\section{Basic equations}

We consider the steady laminar boundary layer of a viscous incompressible fluid over a long thin longitudinal circular cylinder of radius $r_{0}$, moving with a constant velocity $u_{w}$ in parallel or reversely to a free stream of uniform velocity $u_{\infty}$, as shown in Fig. 1 . Either the surface velocity or the free stream velocity may be zero but not both. It is assumed that the surface temperature of the cylinder $T_{w}$ and the ambient fluid temperature $T_{\infty}$ are taken to be constant, where $T_{w}>T_{\infty}$. It is also assumed that the radius of the cylinder is larger compared with the boundary layer thickness, so that the boundary layer curvature can be neglected. Under such assumptions and neglecting the viscous dissipation, the boundary layer equations are

$$
\begin{aligned}
& \frac{\partial(r u)}{\partial x}+\frac{\partial(r v)}{\partial r}=0 \\
& u \frac{\partial u}{\partial x}+v \frac{\partial u}{\partial r}=\frac{v}{r} \frac{\partial}{\partial r}\left\{r \frac{\partial u}{\partial r}\right\} \\
& u \frac{\partial T}{\partial x}+v \frac{\partial T}{\partial r}=\frac{1}{\operatorname{Pr}} \frac{1}{r} \frac{\partial}{\partial r}\left\{r \frac{\partial T}{\partial r}\right\}
\end{aligned}
$$



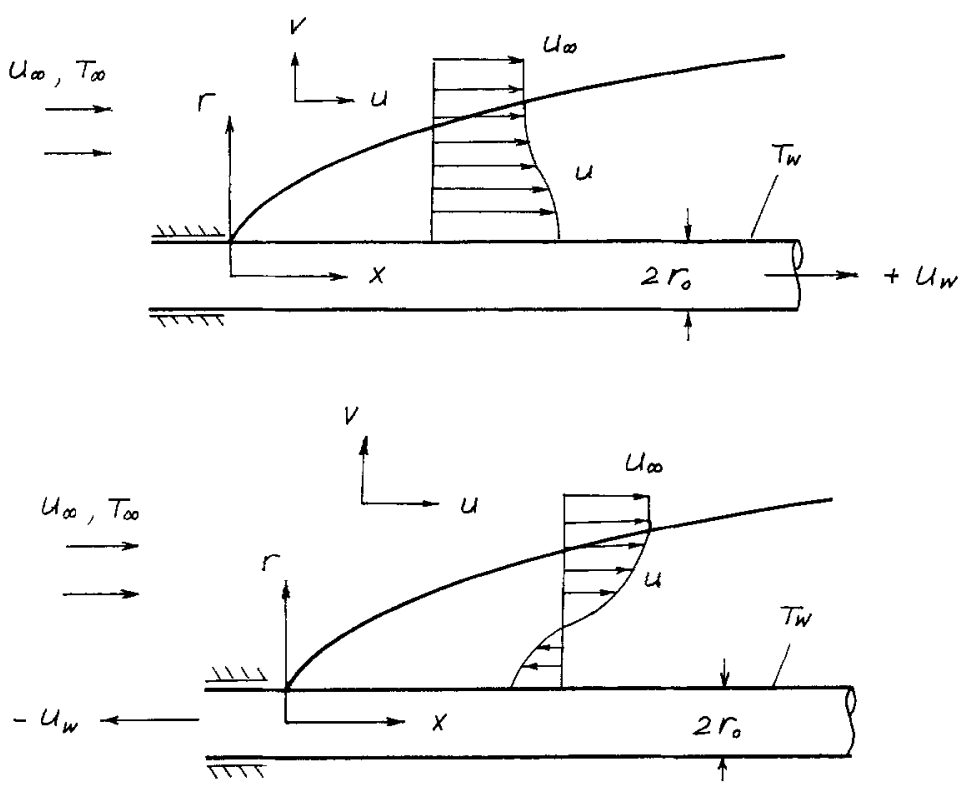

Fig. 1. Physical model and coordinate system

with the boundary conditions

$$
\begin{aligned}
& r=r_{0}: u= \pm u_{w}, \quad v=0, \quad T=T_{w} \\
& r=\infty: u=u_{\infty}, \quad T=T_{\infty} .
\end{aligned}
$$

Here $x$ and $r$ are coordinates along the axis of the cylinder and normal to it, $u$ and $v$ are the velocity components along $x$ - and $r$-directions, $T$ is the temperature of the fluid, $v$ is the kinematic viscosity and Pr is the Prandtl number. The boundary condition of $u=+u_{w}$ in Eq. (4) represents the case of a cylinder moving in parallel to the free stream, whilst $u=-u_{w}$ represents the case of a cylinder moving reversely to it, respectively.

It is known that due to the curvature effect of the cylinder, no similarity solutions of Eqs. (1) to (5) are available. In order to analyze the effect of both the moving cylinder and the free stream on the boundary layer flow, we introduce the following variables, similar to those suggested by Lin and Huang [18] for the corresponding problem of a flat plate. Let us first define

$$
\begin{aligned}
& \bar{x}=4 \frac{\frac{x}{r_{0}}}{\operatorname{Re}_{w}+\operatorname{Re}_{\infty}} ; \quad \bar{y}=\frac{r^{2}-r_{0}{ }^{2}}{r_{0}{ }^{2}} ; \quad \bar{\psi}=\frac{2 \psi}{v r_{0}\left(\operatorname{Re}_{w}+\operatorname{Re}_{\infty}\right)} ; \quad \theta=\frac{T-T_{\infty}}{T_{w}-T_{\infty}} \\
& \xi=\bar{x} ; \quad \eta=\frac{\bar{y}}{\sqrt{\bar{x}}} ; \quad f(\xi, \eta)=\frac{\bar{\psi}}{\sqrt{\bar{x}}} ; \quad g(\xi, \eta)=\theta
\end{aligned}
$$

where the stream function, $\psi$, is defined by

$$
r u=\frac{\partial \psi}{\partial y} ; \quad r v=-\frac{\partial \psi}{\partial x}
$$


Equations (1)-(3) are then transformed to

$$
\begin{aligned}
& \left\{(1+\xi \eta) f^{\prime \prime}\right\}^{\prime}+\frac{1}{2} f f^{\prime \prime}=\xi\left\{f^{\prime} \frac{\partial f^{\prime}}{\partial \xi}-f^{\prime \prime} \frac{\partial f}{\partial \xi}\right\} \\
& \frac{1}{\operatorname{Pr}}\left\{(1+\xi \eta) g^{\prime}\right\}^{\prime}+\frac{1}{2} f g^{\prime}=\xi\left\{f^{\prime} \frac{\partial g}{\partial \xi}-g^{\prime} \frac{\partial f}{\partial \xi}\right\}
\end{aligned}
$$

subject to he boundary conditions

$\eta=0: f^{\prime}(\xi, 0)= \pm \lambda, \quad f(\xi, 0)=0 ; \quad g(\xi, 0)=1$

$\eta=\infty: f^{\prime}(\xi, \infty)=1 \mp \lambda ; \quad g(\xi, \infty)=0$

which can be reduced to the conventional boundary conditions for a static and a moving cylinder by setting $\lambda=0$ and 1 , respectively.

The quantities of main physical interest are the skin friction and the heat transfer from the cylinder, which are given by

$\tau_{w}=\mu\left|\left\{\frac{\partial u}{\partial r}\right\}_{r=r_{0}}\right|$

and

$q_{w}=-k\left\{\frac{\partial T}{\partial r}\right\}_{r=r_{0}}$

where $\mu$ and $k$ are the dynamic viscosity and thermal conductivity of the fluid. The coefficients of skin friction for a static and a moving cylinder are defined as

$C_{f \infty}=\frac{\tau_{w}}{\varrho u_{\infty}^{2}}$

and

$C_{f w}=\frac{\tau_{w}}{\varrho u_{w}^{2}}$.

Also, the local Nusselt number is given by

$N u=\frac{x q_{w}}{k\left(T_{w}-T_{\infty}\right)}$.

In terms of the transformed variables, we get

$C_{f \infty} \operatorname{Re}_{\infty x}^{1 / 2}=\frac{f^{\prime \prime}(\xi, 0)}{(1-\lambda)^{3 / 2}}$

where $\operatorname{Re}_{\infty \infty x}=u_{\infty} x / v$ and

$C_{f w} \operatorname{Re}_{w x}^{1 / 2}=\frac{f^{\prime \prime}(\xi, 0)}{\lambda^{3 / 2}}$ 
where $\operatorname{Re}_{w x}=u_{w} x / v$. Also, we get

$\frac{\mathrm{Nu}}{\sqrt{\operatorname{Re}_{\infty x}}}=-\frac{g^{\prime}(\xi, 0)}{\sqrt{1-\lambda}}$

$\frac{\mathrm{Nu}}{\sqrt{\mathrm{Re}_{w x}}}=-\frac{g^{\prime}(\xi, 0)}{\sqrt{\lambda}}$

It should be noted that Eqs. (17) and (19) are for any value of $\lambda$ except $\lambda=1$, whilst Eqs. (18) and (20) are for moving cylinders except $\lambda=0$.

\section{Results and discussion}

A finite-difference method known as Keller's box method proposed by Keller and Cebeci [17] with Newton's linearization method has been used to solve the parabolic differential equations (8) and (9) with the associated boundary conditions (10) and (11). Since a good description of this method and its application to some boundary layer problems are given in [19] - [21], it will not be presented here.

The accuracy of the predictive results has been established by comparison with known results. Tables 1 and 2 compare the local Nusselt number data with the results of Lin and Shih [9] obtained by the method of local similarity solutions. The agreement between these results is excellent.

Representative velocity profiles and temperature distributions versus $\eta$ are shown in Figs. 2 to 9 , exhibiting the effects of the curvature parameter $\xi$, Prandtl number Pr and relative velocity

Table 1. Comparison of $-g^{\prime}(\xi, 0)$ for a static cylinder $(\lambda=0)$

\begin{tabular}{lllllll}
\hline Pr & \multicolumn{2}{c}{72} & \multicolumn{2}{c}{10.} & \multicolumn{2}{c}{100.} \\
\hline$\xi$ & Present & $\begin{array}{l}\text { Lin and } \\
\text { Shih [9] }\end{array}$ & Present & $\begin{array}{l}\text { Lin and } \\
\text { Shih [9] }\end{array}$ & Present & $\begin{array}{l}\text { Lin and } \\
\text { Shih [9] }\end{array}$ \\
\hline .05 & .5997 & .60494 & 1.4742 & 1.47596 & 3.1805 & 3.17525 \\
.1 & .6153 & .61842 & 1.4984 & 1.49537 & 3.2214 & 3.20628 \\
.5 & .7354 & .71996 & 1.6784 & 1.64073 & 3.5172 & 3.43493 \\
1.0 & .8678 & .83568 & 1.8734 & 1.80445 & 3.8280 & 3.68680 \\
1.5 & .9887 & .94358 & 2.0486 & 1.95518 & 4.1001 & 3.91357 \\
\hline
\end{tabular}

Table 2. Comparison of $-g^{\prime}(\xi, 0)$ for a moving cylinder in parallel flow $(\lambda=1)$

\begin{tabular}{lrrrrrr}
\hline Pr & \multicolumn{2}{c}{.72} & \multicolumn{2}{c}{10.} & \multicolumn{2}{c}{100.} \\
\hline$\xi$ & Present & $\begin{array}{c}\text { Lin and } \\
\text { Shih [9] }\end{array}$ & Present & $\begin{array}{l}\text { Lin and } \\
\text { Shih [9] }\end{array}$ & Present & $\begin{array}{l}\text { Lin and } \\
\text { Shih [9] }\end{array}$ \\
\hline .05 & .6991 & .71948 & 3.3574 & 3.36934 & 11.1003 & 11.09821 \\
.1 & .7112 & .72692 & 3.3626 & 3.37813 & 11.1105 & 11.10713 \\
.5 & .7875 & .78852 & 3.4506 & 3.44792 & 11.1941 & 11.17821 \\
1.0 & .8749 & .86611 & 3.5508 & 3.53217 & 11.2964 & 11.26498 \\
1.5 & .9593 & .95695 & 3.6499 & 3.61983 & 11.3988 & 11.35507 \\
2.0 & 1.0411 & 1.03830 & 3.7481 & 3.70241 & 11.5013 & 11.44147 \\
\hline
\end{tabular}




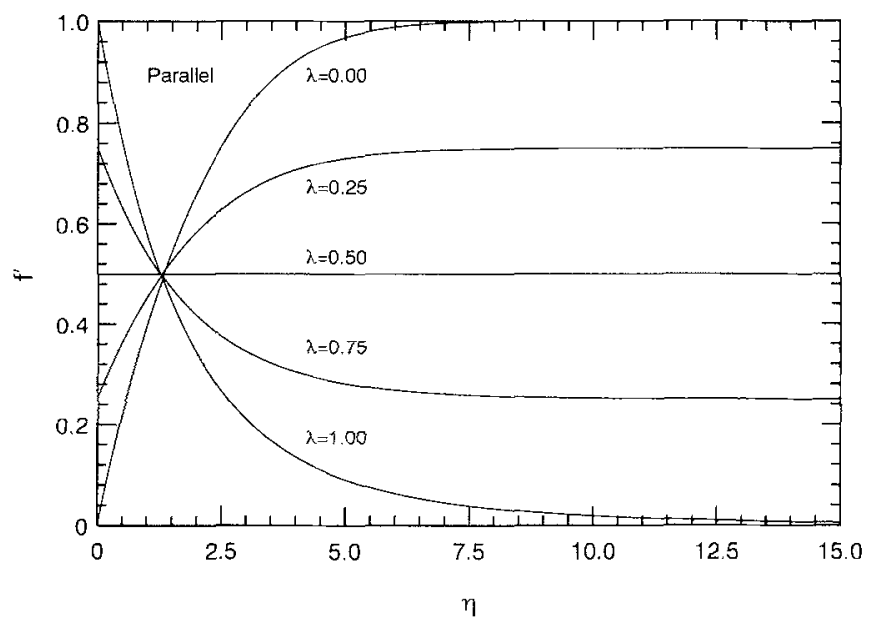

Fig. 2. Velocity profiles $f^{\prime}$ versus $\eta$ for $\xi=0.5$; parallel moving cylinder

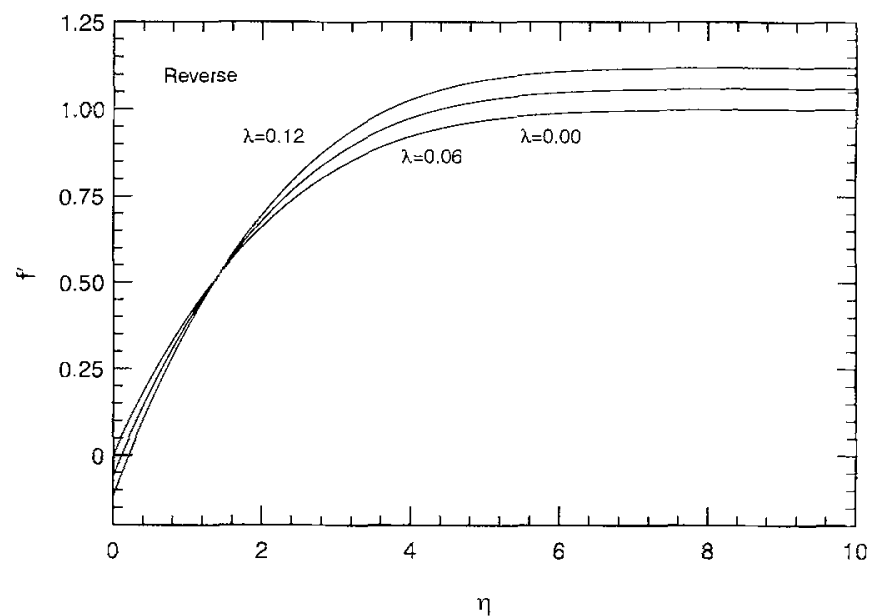

Fig. 3. Velocity profiles $f^{\prime}$ versus $\eta$ for $\xi=0.5$; reverse moving cylinder

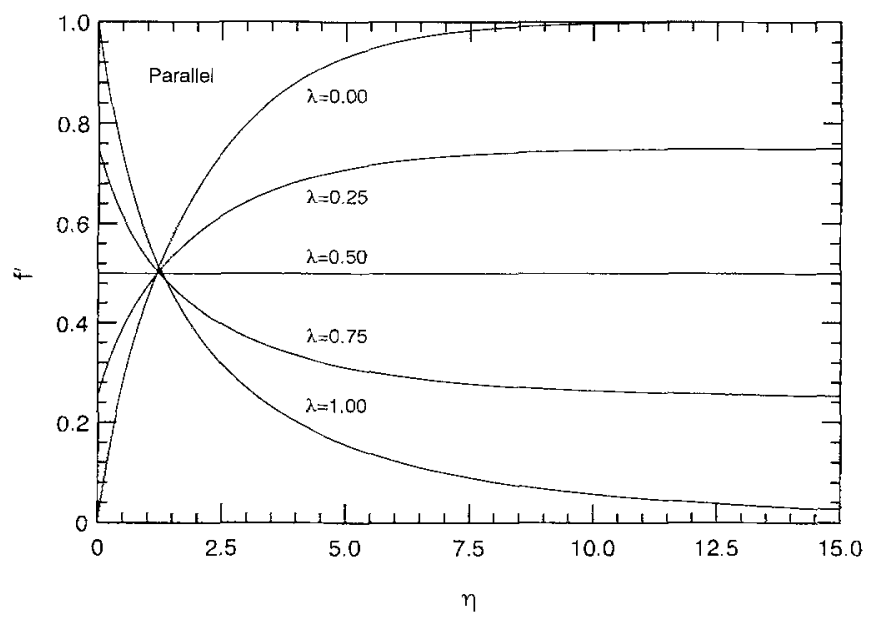

Fig. 4. Velocity profiles $f^{\prime}$ versus $\eta$ for $\xi=1.5$; parallel moving cylinder 
Heat transfer over a longitudinal circular cylinder

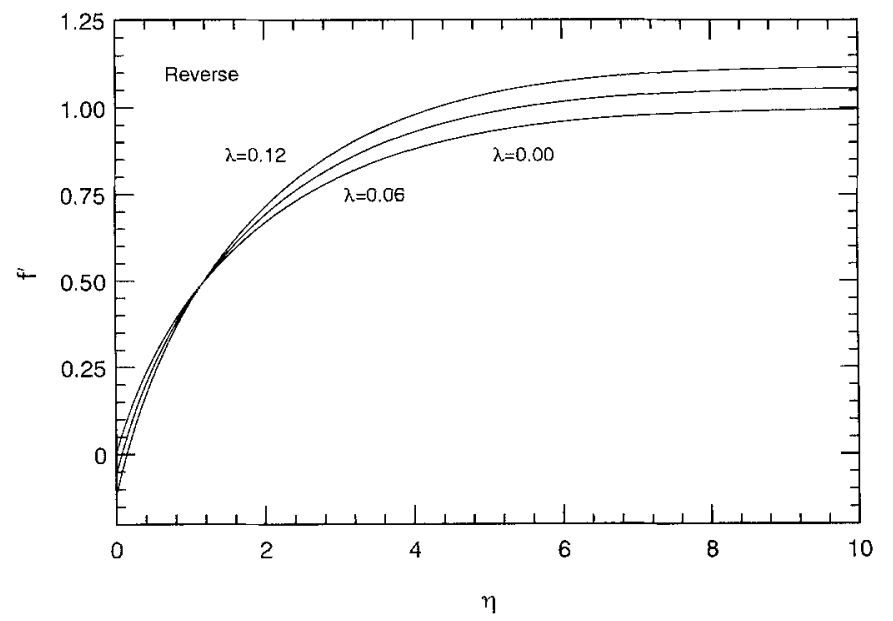

Fig. 5. Velocity profiles $f^{\prime}$ versus $\eta$ for $\xi=1.5$; reverse moving cylinder

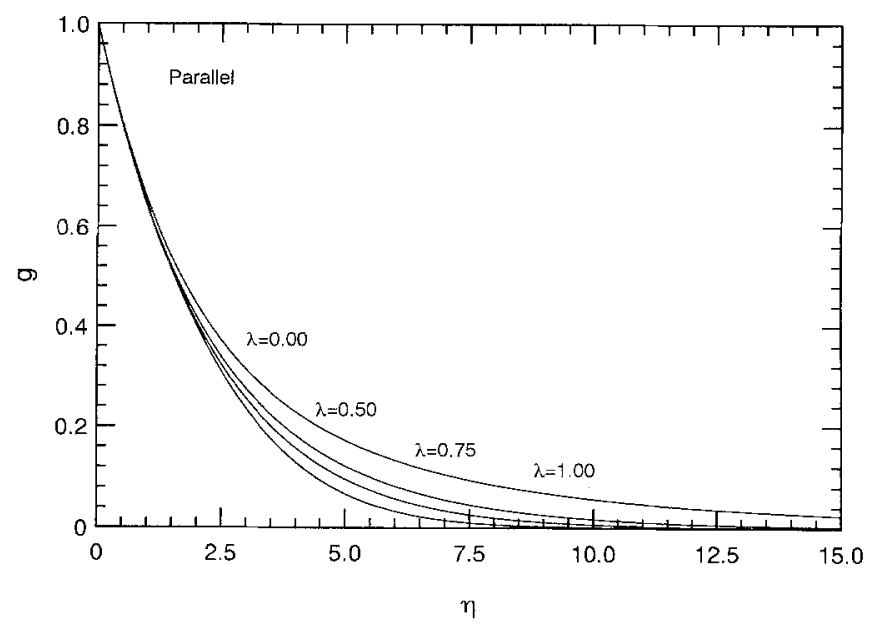

Fig. 6. Temperature profiles $g$ versus $\eta$ for $\xi=0.5$ and $\operatorname{Pr}=0.72$; parallel moving cylinder

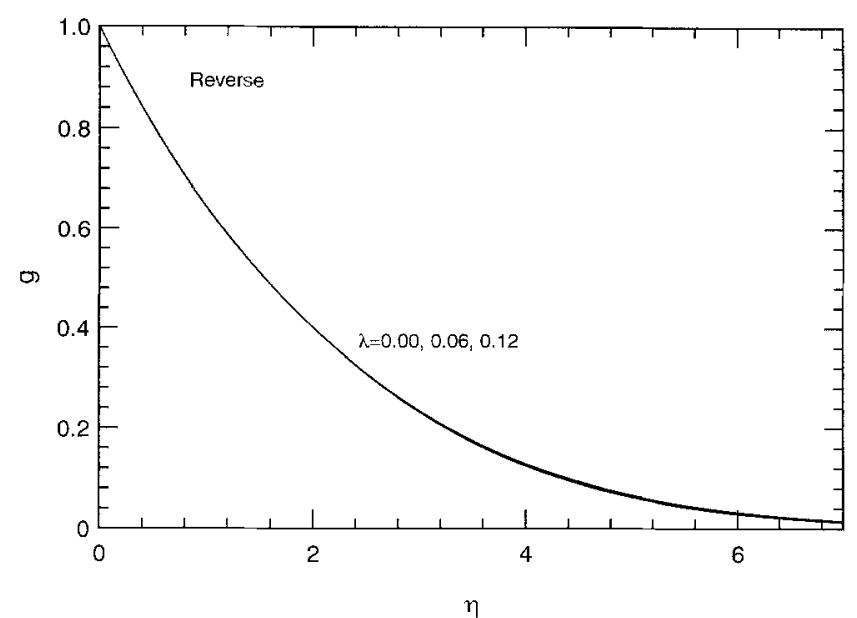

Fig. 7. Temperature profiles $g$ versus $\eta$ for $\xi=0.5$ and $\operatorname{Pr}=0.72$; reverse moving cylinder 


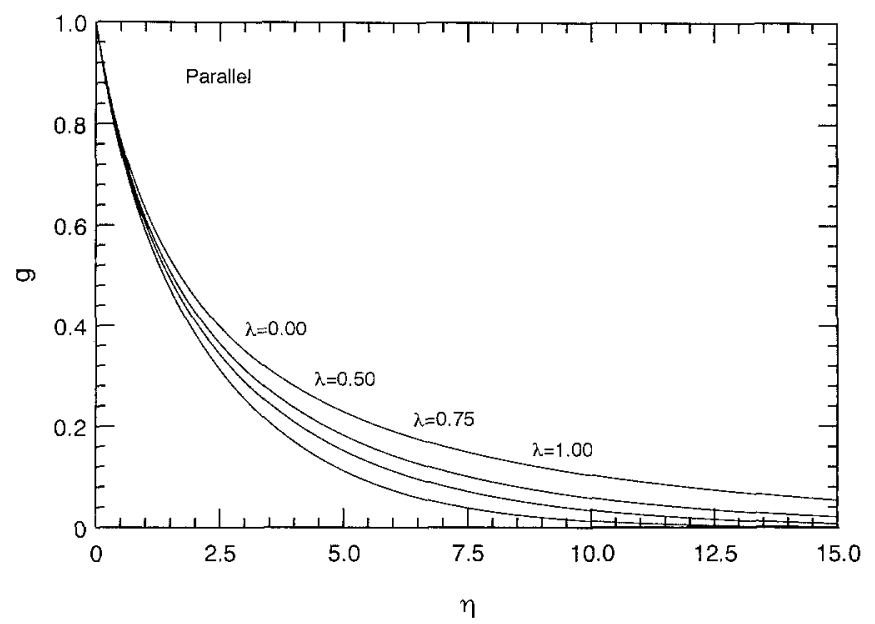

Fig. 8. Temperature profiles $g$ versus $\eta$ for $\xi=1.5$ and $\operatorname{Pr}=0.72$; parallel moving cylinder

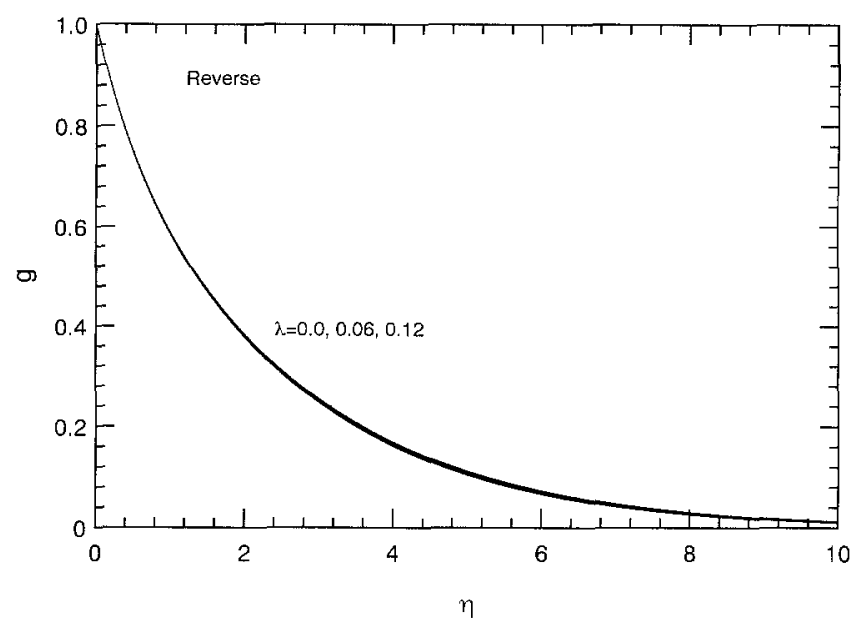

Fig. 9. Temperature profiles $\mathrm{g}$ versus $\eta$ for $\xi=1.5$ and $\mathrm{Pr}=0.72$; reverse moving cylinder

parameter $\lambda$. Figures 2 and 4 show clearly a gradual conversion of the velocity profiles as the relative velocity parameter $\lambda$ increases from 0 to 1 . On the other hand, it is seen from Figs. 3 and 5 that the velocity profiles first decrease as the parameter $\lambda$ increases, followed by an increase as a cross-over point is passed, when the cylinder is moving reversely to the free stream. A reverse flow region near the surface of the cylinder (at the region of small $\eta$ ) can also be seen in these figures.

The non-dimensional temperature profiles are shown in Figs. 6 to 9 for the curvature parameter $\xi$ of $\xi=0.5$ and 1.5 when $\operatorname{Pr}=0.72$ (air). As can be seen from these figures, the fluid temperature increases with the increase of $\lambda$ when the cylinder is moving parallel to the free stream. However, the difference in temperature profiles is very small for the range of $\lambda$ for the case of a cylinder moving reversely to the free stream.

The variation of the skin friction coefficients is shown in Fig. 10. We notice that when the cylinder is moving in parallel to the free stream, $C_{f \infty} \sqrt{\mathrm{Re}_{\infty x}}$ decreases from $0.7342,0.4893$, and 0.3306 , respectively, to zero for $\xi=1.5,0.5$, and 0.0 (flat plate) as $\lambda$ increases from 0 to 0.5 , 
Heat transfer over a longitudinal circular cylinder

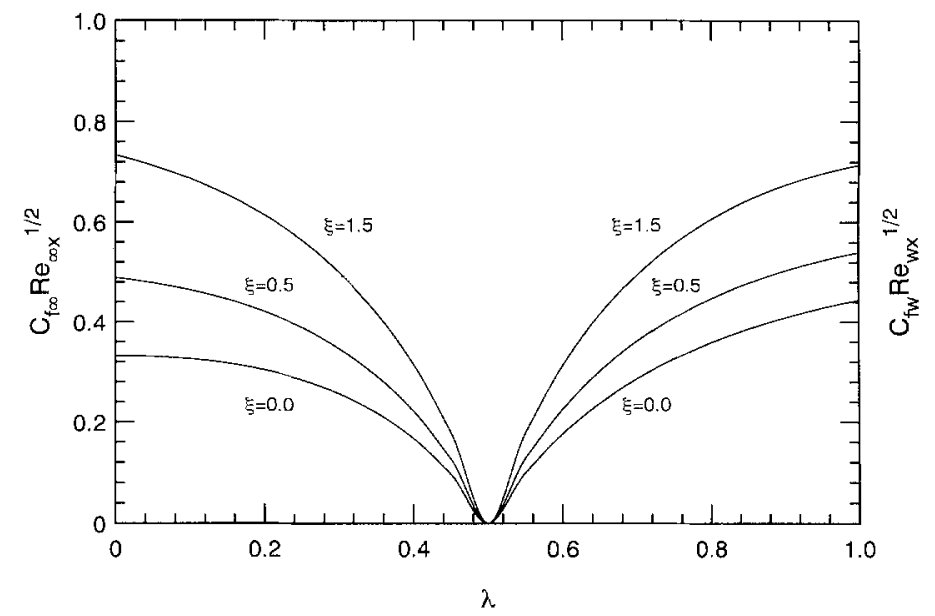

Fig. 10. Variation of the local skin friction coefficients $C_{f \infty} \sqrt{\operatorname{Re}_{\infty x}}$ and $C_{f_{w}} \sqrt{\operatorname{Re}_{w x}}$ versus $\lambda$ for $\xi=0$ (flat plate), $\xi=0.5$ and $\xi=1.5$

Table 3. Coefficient of skin friction $C_{f \infty} \sqrt{\mathrm{Re}_{\infty x}}$ for reverse flow case

\begin{tabular}{lllllll}
\hline$\lambda$ & $\xi=0$ & $\xi=.3$ & $\xi=.6$ & $\xi=.9$ & $\xi=1.2$ & $\xi=1.5$ \\
\hline .00 & .33206 & .43122 & .51675 & .59421 & .66621 & .73418 \\
.02 & .33186 & .43355 & .52072 & .59946 & .67253 & .74146 \\
.06 & .33022 & .43708 & .52739 & .60852 & .68360 & .75430 \\
.10 & .32713 & .43932 & .53261 & .61592 & .69280 & .76505 \\
.11 & .32614 & .43970 & .53371 & .61753 & .69481 & .76736 \\
.12 & .32507 & .44002 & .53473 & .61904 & .69603 & .76924 \\
.13 & .32392 & .44027 & .53567 & .62041 & .68910 & \\
.14 & .32269 & .44047 & .53653 & .62157 & & \\
.15 & .32139 & .44060 & .53728 & & & \\
.16 & .32000 & .44067 & .53789 & & & \\
.17 & .31854 & .44069 & & & & \\
.18 & .31701 & & & & & \\
\hline
\end{tabular}

Table 4. Local Nusselt number $\mathrm{Nu} / \sqrt{\operatorname{Re}_{\infty x}}$ for reverse flow case when $\operatorname{Pr}=0.72$

\begin{tabular}{lllllll}
\hline$\lambda$ & $\xi=0$ & $\xi=.3$ & $\xi=.6$ & $\xi=.9$ & $\xi=1.2$ & $\xi=1.5$ \\
\hline .00 & .29564 & .38179 & .46266 & .53632 & .60500 & .66999 \\
.02 & .29041 & .37709 & .45782 & .53118 & .59950 & .66411 \\
.06 & .28015 & .36806 & .44854 & .52135 & .58899 & .65287 \\
.10 & .27008 & .35947 & .43978 & .51206 & .57906 & .64223 \\
.11 & .26759 & .35739 & .43766 & .50982 & .57664 & .63959 \\
.12 & .26510 & .35534 & .43557 & .50759 & .57419 & \\
.13 & .26263 & .35331 & .43350 & .50535 & & \\
.14 & .26016 & .35130 & .43144 & & & \\
.15 & .25769 & .34932 & .42938 & & & \\
.16 & .25523 & .34735 & .42730 & & & \\
.17 & .25277 & .34541 & & & & \\
.18 & .25032 & & & & & \\
\hline
\end{tabular}


respectively. On the other hand, $C_{f w} \sqrt{\mathrm{Re}_{w x}}$ increases from zero to $0.7136,0.5394$ and 0.4437 , respectively, for $\xi=1.5,0.5$ and 0.0 (flat plate) as $\lambda$ increases from 0.5 to 1.0 .

Representative values of the skin friction coefficient $C_{f \infty} \sqrt{\operatorname{Re}_{\infty x}}$ and local Nusselt number $\mathrm{Nu} \sqrt{\operatorname{Re}_{\infty x}}$ corresponding to a cylinder moving reversely to the free stream are given in Tables 3 and 4 for some specific values of $\lambda$ and $\xi$ when $\operatorname{Pr}=0.72$. It is seen that $C_{f \infty} \sqrt{\operatorname{Re}_{\infty} x}$ increases, while $\mathrm{Nu} \sqrt{\mathrm{Re}_{\infty x}}$ decreases as $\lambda$ increases. Further, they increase with the increase of the curvature parameter $\xi$. The numerical results also indicate that for some values of $\lambda$ and $\xi$ the laminar boundary layer breaks down due to the opposite moving of the cylinder and the free stream. Therefore, the convergent numerical solutions cannot be obtained beyond the values of $\lambda$ and $\xi$ indicated in Tables 3 and 4 .

\section{References}

[1] Seban, R. A., Bond, R.: Skin friction and heat transfer characteristics of a laminar boundary layer on a circular cylinder in axial incompressible flow. J. Aero. Sci. 18, $671-675$ (1951).

[2] Kelly, H. R.: A Note on the laminar boundary layer on a circular cylinder in axial incompressible flow. J. Aero. Sci. 21, 634 (1962).

[3] Glauert, M. B.: The axisymmetric boundary layer on a long cylinder. Proc. R. Soc London Ser. A 230, $189-203(1954)$.

[4] Stewartson, K.: The asymptotic boundary layer on a circular cylinder in axial incompressible flow. Q. Appl. Math. 13, 113-122 (1955).

[5] Wanous, P. J., Sparrow, E. M.: Longitudinal flow over a circular cylinder with surface mass transfer. A.I.A.A. J. 3, $147-149$ (1965).

[6] Jaffe, N. A., Okamura, T. T.: The transverse curvature effect of the incompressible boundary layer for longitudinal flow over a cylinder. ZAMP 19, 564-574 (1968).

[7] Cebeci, T.: Laminar and turbulent incompressible boundary layers on slender bodies of revolution in axial flow. J. Basic Eng. 92, 545-553 (1970).

[8] Curle, S. N.: Calculation of the axisymmetric boundary layer on a long thin cylinder. Proc. R. Soc. London Ser. A 372, 555- 564 (1979).

[9] Lin, H. T., Shih, Y. P.: Laminar boundary layer heat transfer along static and moving cylinders. J. Chin. Inst. Eng. 3, $73-79$ (1980).

[10] Sawchuk., S. P., Zamir, M.: Boundary layer on a circular cylinder in axial flow. Int. J. Heat Fluid Flow 13, $184-188$ (1992).

[11] Sakiadis, B. C.: Boundary layer behavior on continuous solid surfaces. III. The boundary layer on a continuous cylindrical surface. A.I.Ch.E. J. 7, 467-472 (1961).

[12] Rotte, J. W., Beek, W. J.: Some models for the calculation of heat transfer coefficients to a moving continuous cylinder. Chem. Eng. Sci. 24, 705-716 (1969).

[13] Karnis, J., Pechoc, V.: The thermal laminar boundary layer on a continuous cylinder. Int. J. Heat Mass Transfer 21, 43-47 (1978).

[14] Choi, I. G.: The effect of variable properties of air on the boundary layer for a moving continuous cylinder. Int. J. Heat Mass Transfer 25, 597-602 (1982).

[15] Pop, I., Kumari, M., Nath, G.: Non-Newtonian boundary layers on a moving cylinder. Int. J. Eng. Sci. 28, $303-312$ (1990).

[16] Eswara, A. T., Nath, G.: Unsteady forced convection laminar boundary layer flow over a moving longitudinal cylinder. Acta Mech. 93, 13-28 (1992).

[17] Keller, H. B., Cebeci, T.: Accurate numerical methods for boundary layers. Part I. Two-dimensional laminar flows. In: Proc. 2nd Int. Conf. Numerical Methods in Fluid Dynamics. Lecture Notes in Physics, Vol. 8. New York: Springer 1971.

[18] Lin, H. T., Huang, S. F.: Flow and heat transfer of plane surfaces moving in parallel and reversely to the free stream. Int. J. Heat Mass Transfer 37, $333-336$ (1994).

[19] Na, T. Y.: Numerical solution of natural convection flows past a non-isothermal vertical flat plate. Appl. Sci. Res. 33, 519-543 (1978). 
Heat transfer over a longitudinal circular cylinder

[20] Na, T. Y.: Computational methods in engineering boundary value problems. New York: Academic Press 1979.

[21] Na, T. Y., Pop, I.: Free convection flow past a vertical flat plate embedded in a saturated porous medium. Int. J. Eng. Sci. 21, 517-526 (1983).

Authors' addresses: Prof. T. Y. Na, Department of Mechanical Engineering, University of MichiganDearborn, Dearborn, MI, 48128-1491, U.S.A. and Prof. I. Pop, Faculty of Mathematics, University of Cluj, R - 3400 Cluj, CP 253, Romania 